\title{
PREFACE: A SPECIAL ISSUE CELEBRATING A NEW UQ ACTIVITY GROUP IN CHINA
}

Uncertainty quantification (UQ) has been an important research topic in computational sciences and scientific computing for many years. The aim of this special issue is to celebrate a newly founded UQ activity group under the China Society for Industrial and Applied Mathematics (CSIAM). Founded in 1990, CSIAM is one of the biggest societies for Chinese applied mathematicians. Currently, CSIAM has more than 12,000 regular members. CSIAM aims to bridge mathematics and the industrial and business communities and to promote cooperation between mathematicians and engineers, as well as corporate managers. It also aims to solve various mathematical problems hindering economic and technological development, and to further research and education in applied mathematics. The current president of CSIAM is Professor Pingwen Zhang from Peking University.

The new UQ activity group aims at enhancing activity and collaboration on all aspects of the effects of uncertainty on mathematical descriptions of real phenomena. It serves to support interactions among mathematicians, statisticians, engineers, and scientists working in the interface of computation, analysis, and probability. The founding chair of the UQ activity group is Professor Tao Tang from Southern University of Sciences and Technology.

There are 11 papers in this special issue, all of which were selected after a careful and studious peer-review process. Those papers cover various important topics in UQ, for instance, model reduction, sparse polynomial approximations, nonlinear filters, oilfield simulations, surrogate modeling for Bayesian inverse problems, variance reduction, and parametric regularity analysis, to name a few.

Finally, we would like to express our sincere thanks to all the authors and the referees for their contributions to this special issue for the International Journal for Uncertainty Quantification. Special thanks go to Professor Habib N. Najm (Editor-in-Chief of IJUQ) for providing the chance to organize this special issue and Mr. Brandon T. Bisceglia (IJUQ Editorial Office) for his professional and patient help and instructions during the preparation of this issue. We also would like to thank Professor Nicholas Zabaras (Founding Editor-in-Chief of IJUQ) for his encouragement and support for organizing this special issue. We sincerely hope our readers will like and enjoy all the articles in the special issue.

Guest Editor:

Tao Zhou

Associate Editor of IJUQ

Vice Chair and Secretary, CSIAM UQ Activity Group

Chinese Academy of Sciences 